\title{
La orientación educativa en las prácticas inclusivas de la educación superior cubana
}

\author{
Elsie Alejandrina Pérez Serrano \\ Universidad de Holguín - Holguín - Cuba \\ Elsa María Hernández Ochoa \\ Universidad de Cienfuegos - Cienfuegos - Cuba
}

\begin{abstract}
Resumen
El artículo forma parte de un estudio sobre la inclusión en la educación superior en Cuba y su objetivo es describir las prácticas de orientación educativa a los alumnos con necesidades educativas especiales o que se encuentran en situación de vulnerabilidad. Se realiza un estudio de casos en las universidades de Holguín y Cienfuegos. El resultado muestra que la orientación educativa auxilia los procesos formativos mediante la ayuda a los profesores, para la atención a la diversidad del alumnado; a los alumnos, en la organización de la actividad de aprendizaje, la resolución de problemas y los proyectos de vida y, a los padres, para apoyar sus hijos en la toma de decisiones. El estudio evidencia que la articulación de postulados de la psicología histórico cultural, la pedagogía sobre las necesidades educativas especiales y el modelo social de la discapacidad, constituye un fundamento congruente con la inclusión en la educación superior.
\end{abstract}

Palabras clave: Orientación educativa; educación inclusiva; educación superior.

\section{The educational orientation in the inclusive practices of Cuban Higher Education}

\begin{abstract}
This article is part of a study on the inclusion processes in the Higher Education in Cuba and its objective is to show the good practices of educational orientation to the students with Special Needs or some vulnerability situation. A case study is carried out at the universities of Holguín and Cienfuegos. As a result is obtained that the educational orientation is one of the components that aids the formative processes through the help to the professors, get supports in the attention to the diversity of the students on the organization of the learning activity, there solution of problems and the projects of life and to the parents so that they support their children in the taking of decisions. The study evidences that the articulation of postulates of historical cultural psychology, the pedagogic conception about the special educational necessities and of the social pattern of the Special Needs, it constitutes an appropriate basics with the purposes of the inclusion in the Higher Education.
\end{abstract}

Keywords: Education guidance; inclusive education; Higher Education.

\section{A orientação educativa nas práticas inclusivas da educação superior cubana}

\section{Resumo}

Este artigo faz parte de um estudo sobre a inclusão na educação superior em Cuba e seu objetivo é descrever as práticas de orientação educativa aos alunos com necessidades educativas especiais ou que se encontram em situação de vulnerabilidade. Realizou-se um estudo de caso nas universidades de Holguín e Cienfuegos. O resultado mostra que a orientação educativa auxilia os processos formativos mediante a ajuda aos professores, para a atenção à diversidade do alunado; aos alunos, na organização da atividade de aprendizagem, a resolução de problemas e os projetos de vida e, aos pais, para apoiar seus filhos na tomada de decisões. O estudo evidencia que a articulação de postulados da psicologia histórico-cultural, a pedagogia sobre as necessidades educativas especiais e o modelo social da deficiência constitui um fundamento congruente com a inclusão na educação superior.

Palavras-chave: Orientação educativa; educação inclusiva; ensino superior. 


\section{Introducción}

La educación inclusiva avanza a ritmo desigual en los diferentes países irrumpiendo paulatinamente en la educación superior. Con ello, comienzan a declinar las prácticas arraigadas en la universidad tradicional $y$, aumentan las oportunidades para el alumnado que, por diferentes factores, no accedía a la educación superior. Estos alumnos tienen variadas demandas para el aprendizaje y el desarrollo, las que se designan con el concepto de necesidad educativa especial, el cual enfatiza en las ayudas para transitar con éxito por la educación.

En Cuba la educación es un derecho para todos, puesto que "una sociedad inclusiva sólo puede ser el resultado de la política de un Estado en su conjunto" (Unesco, 2008 , p.8), lo cual se refleja en la abolición del analfabetismo en 1961 y disminución de la tasa al 3,9 \% (Pérez-Cruz, 2011). En la actualidad la población no alfabetizada es del 0,2\% (Cuba, 2014).

La Conferencia internacional de educación, La educación inclusiva: el camino hacia el futuro (Unesco, 2008) presenta de forma asequible fundamentos de políticas que coadyuvan a imbuir a los docentes, en procesos de acompañamiento a la diversidad del alumnado. Aunque, como es frecuente en las normativas enfocadas al tema, se enmarca en la educación básica. De manera, que el marco legal para la educación superior es incipiente y está arraigada la idea de que es para un alumnado selecto, lo cual no favorece la configuración de recursos pedagógicos para satisfacer demandasen este nivel de educación.

Los logros de Cuba en la educación primaria y media se reflejan en el Índice de Desarrollo de la Educación para Todos (IDE) con el lugar 28, siendo el país latinoamericano mejor ubicado (Unesco, 2015). El acceso universal a la universidad es una realidad, por ejemplo, el $60 \%$ de la población de entre 18 y 24 años se encuentra realizando estudios superiores (Cuba, 2014), no obstante, los procesos de inclusión en este nivel no adquieren la relevancia que poseen en los niveles educativos anteriores y son más espontáneos.

El país cumplió los Objetivos de Desarrollo del Milenio y acoge el documento Transformar nuestro mundo: la Agenda 2030 para el Desarrollo Sostenible, que enuncia 17 objetivos de desarrollo sostenible y 169 metas conexas. El objetivo cuatro proyecta un modelo nuevo de universidad cuando formula "Garantizar una educación inclusiva, equitativa y de calidad y promover oportunidades de aprendizaje durante toda la vida para todos." (ONU, 2015, p.16)

A pesar de los logros educacionales el sistema de acceso a la educación superior es el mismo para todos los aspirantes, lo que coloca en desventaja a los que tienen necesidades educativas especiales. Solamente se dispone de la circular: Sobre el tratamiento a los jóvenes discapacitados para estudiar en la Educación Superior (Cuba, 2010) que norma ayudas para la realización de los exámenes de ingreso e indicaciones preferenciales de acceso a las carreras. Sin embargo, el alumno con discapacidad no es el único que requiere de ayudas para ingresar a la universidad y culmi- narla con éxito, por lo cual queda a las estructuras metodológicas y organizativas, implementar acciones cuando estos jóvenes arriban a una institución de educación superior.

Una de las vías utilizadas es la orientación educativa, servicio que se encuentra en las residencias estudiantiles e incorpora a los departamentos docentes de las ciencias humanas, particularmente psicología, psicopedagogía y logopedia y educación especial. Por último, y configurando todo un tejido de apoyo se encuentran las estructuras metodológicas, lideradas por los profesores de más experiencia, cuyas funciones tienen en su centro la formación del estudiante.

La primera institución de educación superior creada en Cuba es la Real y Pontificia Universidad de San Gerónimo de la Habana (1728), desde 1899, Universidad de la Habana. Posteriormente, surgen la Universidad de Oriente (1947), la Universidad Central de las Villas (1952) y la Universidad de Camagüey (1975).Con la fundación del Ministerio de Educación Superior (1976) ocurre un proceso de crecimiento y perfeccionamiento con tres hitos relevantes: el surgimiento de universidades, institutos, escuelas, colegios y academias 3/4 con 64 en el 2004 3/4; la universalización con la creación de sedes universitarias en los municipios (2001) y el proceso de integración en cada provincia (2011) disminuyendo a 51 instituciones en el curso 2014-2015 (Domínguez, 2016).

El modelo de formación articula tres dimensiones: instructiva (conocimientos y habilidades), educativa (valores) y desarrolladora (capacidades) y tres componentes: académico (clases), investigativo (trabajo científico) y laboral (práctica). Los estudiantes que no viven en el municipio en que la institución radica tienen residencia estudiantil gratuita. Entre los documentos que norman el trabajo de la educación superior se encuentran vigentes dos reglamentos que instituyen lineamientos esenciales, pero con escasas regulaciones para la inclusión de los alumnos con necesidades educativas especiales.

El primer documento es la Resolución No. $120 / 10$ del Ministerio de Educación Superior o Reglamento de organización docente de la educación superior, constituido por 125 artículos pero solo el 45 establece que las clases de Educación Física se cursarán ante "impedimento físico permanente... en áreas terapéuticas o recibirán clases de ajedrez"(Resolución n 120 /10, 2010, p.13) y los numerados del 61 al 63 contienen "indicaciones generales para las modificaciones al plan del proceso docente de los estudiantes con algún tipo de discapacidad" (Resolución n 120 110, 2010, p.17). En varios capítulos aparecen referencias a cómo proceder con estudiantes por problemas de enfermedad o personales excepcionalmente justificados.

El segundo documento es la Resolución $N^{\circ}$. 132/2004(2004)o Reglamento de Educación de Postgrado de la República de Cuba que, al igual que las Normas y Procedimientos para la Gestión del Postgrado anexas, carece de orientaciones para el ingreso y tránsito exitoso de profesionales con discapacidad por este nivel de formación. La sección Sobre ingreso, convalidación y evaluación no avista especificaciones para los que requieren de apoyos y 
adecuaciones en lo académico, la investigación y el informe de tesis.

Esta carencia se enmienda en la preparación que para cada curso escolar se realiza en las instituciones de educación del país, la cual incluye la orientación educativa como parte de los servicios que se ofrece al alumnado. La orientación educativa se proyecta como una relación de ayuda personal la que, de acuerdo con Calviño (2000), no suplanta al otro en la toma de sus decisiones sino que facilita el reconocimiento de un problema, acompaña en la tarea de ese reconocimiento y asiste en la búsqueda de solución, o sea la orientación:

Se asocia a la idea de facilitar, es convocar, promover la amplitud del análisis, llamar la atención sobre lo que el demandante no está percibiendo, acompañar en una indagación y en una experiencia emocional, significa alternativas de respuesta, no indicación de la solución. (Calviño, 2000, p.17).

La orientación educativa tiene sustento teórico-metodológico general en los postulados de la psicología histórico cultural. En particular en las concepciones de Vigotsky sobre el desarrollo como "complejo proceso dialéctico... de superación de dificultades y de adaptación” (Vigotsky, 1987, p. 96) y, la categoría zona de desarrollo próximo, que posee mayor importancia en la dinámica del desarrollo intelectual y el éxito de la enseñanza que el nivel actual del desarrollo (Vigotsky, 1982). Esta categoría destaca un concepto clave de la teoría: la mediación, que es primordial en los procesos de orientación.

La psicología histórico cultural sostiene que las funciones psíquicas superiores se construyen a lo largo de la historia social del hombre, con soporte biológico en el cerebro y de las que cada ser humano debe apropiarse en su desarrollo ontogenético. El mecanismo psicológico de la apropiación es la internalización que responde a una ley de tránsito de lo social o externo a lo individual e interno, a través de un proceso en el que la mediación a través de signos desarrollados en la vida social, se constituyen en medio de control del propio individuo, de regulación para transformar su comportamiento (Vigostky, 1987).

De manera, que la orientación educativa se erige en un proceso de mediación en el contexto de la actividad de los estudiantes, insertos en un sistema de relaciones sociales, cuyo foco principal es acompañarles en la toma de decisiones. Una parte significativa de la matrícula de las universidades cubanas se encuentra en un período sensitivo del desarrollo, la adolescencia, en el cual se modifican la estructura e interrelaciones de las funciones psíquicas superiores. Estos cambios internos condicionan la influencia del medio sobre el desarrollo psicológico superior (Vigostky, 1987), por lo cual demandan de modalidades de atención que les auxilien en la resolución de los problemas.

Constituyen referencias la obra de Collazo y Puentes (1992), Calviño (2000) y Del Pino y Recarey (2005) sobre orientación educativa y psicológica, la que tiene en cuenta las particularidades del contexto cultural. Sin embargo, disponen de escasas especificidades para la educación superior.

También, se establecen relaciones con la teoría de las necesidades educativas especiales que destaca la interacción entre las demandas del estudiante y la respuesta del entorno. De acuerdo con el modelo social de la discapacidad, la orientación educativa tiene en cuenta la necesidad de superar barreras: políticas (normativas contradictorias), culturales (conceptuales y actitudinales) y didácticas (enseñanza-aprendizaje) (López, 2011).La orientación educativa provee ayuda pedagógica y, al igual que la orientación académica al estudiante con discapacidad en la educación superior, se proyecta con: "uma abordagem holística sobre as habilidades do aluno, o contexto geral da universidade, os serviços de tutoria especializada, de colocação no mercado do trabalho e de estágios, o território e, quando apropriado, a cooperação adequada da família". ${ }^{1}$ (Cabral, Mendes, \& De Anna, 2015, p. 626).

De manera, que mostrar las experiencias en torno a la inclusión de la heterogeneidad del alumnado en la educación superior constituye una vía idónea para visibilizar los avances alcanzados y entrever lo que no se ha conseguido. El objetivo del artículo es mostrar buenas prácticas de orientación educativa a los alumnos con necesidades educativas especiales en las universidades cubanas; a través de un estudio de caso en las universidades de Holguín y Cienfuegos.

\section{Método}

El estudio se realiza con un enfoque dialéctico-materialista respecto a la orientación metodológica de la investigación y la estrategia general en el planteamiento del problema y su solución. Se tiene en cuenta que los objetos, fenómenos y procesos tienen contradicciones internas, en interconexión y unidad indisoluble, que la solución de un problema no descarta el desarrollo anterior y, a su vez, que los cambios cuantitativos originan transformaciones cualitativas esenciales.

La metodología es cualitativa para abordar de forma intensiva, inductiva y a profundidad el objeto luego "consiste en más que un conjunto de técnicas para recoger datos. Es un modo de encarar el mundo empírico" (Taylor y Bogdan, 1996, p.20). Una de las principales dificultades de la metodología cualitativa es la selección de los escenarios, Taylor y Bogdan (1996, p.36) recomiendan "que los investigadores se abstengan de estudiar escenarios en los cuales tengan una directa participación personal o profesional". Sin embargo, la experiencia de más de 20 años como profesoras en las carreras de Educación Especial y Logopedia ofrece una perspectiva axiológica y la sensibilización con la diversidad

1 ...un enfoque holístico de las habilidades del estudiante, el contexto general de la universidad, la tutoría especializada, la ubicación en el mercado de trabajo y las prácticas laborales, el territorio y, cuando corresponde, la cooperación adecuada de la familia. (traducción de las autoras). 
del alumnado en las universidades que no afecta la validez del estudio.

Atendiendo al criterio anterior se realiza un estudio de casos múltiples con muestreo intencional, seleccionados en el curso escolar de septiembre 2015 a julio 2016, para caracterizar la orientación educativa que se realiza en dos contextos: la residencia estudiantil y el proceso de enseñanza y aprendizaje. La estrategia para el estudio de casos sigue las etapas formuladas por Chernousova (2009). La validez de la información se refrenda mediante los criterios de confiabilidad de Guba (Pla, 1999).

Etapa 1. Orientación estratégica, con el objetivo de organizar el rumbo del estudio, y realización de las acciones siguientes:

1. Determinar el objetivo del estudio de caso.

2. Determinar el número de casos a investigar.

3. Determinar el tipo de información a recoger.

4. Seleccionar los informantes para el estudio del caso.

5. Elaborar los instrumentos de investigación y concretar los recursos necesarios.

A continuación una descripción sintética de estas acciones:

Objetivo general: caracterizar la actividad de orientación educativa que se realiza en universidades cubanas para contribuir a la inclusión de los estudiantes con necesidades educativas especiales.

El número de casos seleccionados es de dos: Universidad de Holguín y Universidad de Cienfuegos, en particular, las carreras de Educación Especial y Logopedia.

El proceso de recogida de información se dirige a:

Caracterizar la población que presenta necesidades educativas especiales o se encuentran en condiciones de vulnerabilidad. La información se obtiene a través de observación, revisión de documentos y entrevista.

Caracterizar la orientación educativa que reciben estos alumnos. La información se obtiene a través de revisión de documentos y entrevista (individual y grupal).

Los informantes son: directivos (4), jefes de carrera (4), profesores guías (17), profesores (30), estudiantes (50) de las carreras de Educación Especial y Logopedia de las universidades de Holguín y Cienfuegos, padres (12) y otros profesionales (8).

Recursos: las personas participantes, las instalaciones y equipamientos para desarrollar la orientación y bibliografía sobre el tema.

Etapa 2. Diagnóstico, con el objetivo de aplicar los instrumentos de investigación, para lo cual se realizan las acciones siguientes:

1. Observar el proceso de orientación educativa en diferentes actividades del contexto universitario.
2. Entrevistar a los agentes educativos que participan en la orientación educativa.

3. Estudiar los productos de la actividad de los alumnos.

4. Entrevista semiestructurada a profundidad con el grupo de alumnos.

Etapa 3. Construcción del caso, con el objetivo de integrar los resultados, para lo cual se realizan las acciones siguientes:

1. Estudio longitudinal para el análisis de los datos y la elaboración de cada caso.

2. Estudio transversal para el análisis de los casos y la elaboración del informe.

3. Elaborar las conclusiones del estudio para determinar las principales regularidades.

\section{Resultados}

Caso de estudio: La orientación educativa para la inclusión en universidades cubanas.

Caso 1: La orientación educativa en la Universidad de Holguín.

La Universidad de Holguín (1976) está integrada por 10 facultades, entre ellas la de Educación Infantil, Psicopedagogía y Arte, donde se encuentra el departamento de Educación Especial y Logopedia (1983) integrado por 25 profesores, con doctorado (10), maestría (5) y especialidad (3).La matrícula de la carrera de Educación Especial es de 24 estudiantes (tres árabes) organizados en el primero, cuarto y quinto año. La matrícula de la carrera de Logopedia es de 94 estudiantes de primero a quinto años. Presentan necesidades educativas especiales cuatro estudiantes de la carrera de Educación Especial y 20 de la carrera de Logopedia, o sea 24 estudiantes requieren de una orientación educativa específica para su permanencia y egreso exitoso. (Ver Tabla 1)

Las causas de estas necesidades se relacionan con: debilidad visual por miopía progresiva (1), insuficiencia renal (1), afecciones del corazón (1), fisura bucal (1), desmielinización progresiva (1), problemas motores (1), enfermedades psiquiátricas de tipo neurótico (2) trastorno de personalidad (1), dislalia (6) — cinco por rotacismo y uno con sigmatis$\mathrm{mo}^{3} / 4$, tonopatía por falsete persistente (1), problemas de atención (1) y retardo en el desarrollo psíquico (1).

En condiciones de vulnerabilidad se encuentran seis estudiantes, dadas por dificultades económicas (3) y problemas sociales (3). Los factores económicos se relacionan con bajos ingresos familiares y los de tipo social el alcoholismo. Entre estos últimos, una alumna presenta la situación más desfavorable con una familia desajustada por alcoholismo, enfermedades psiquiátricas (suicidio) y venta de la vivienda. 
Tabla 1. Alumnos con necesidades educativas especiales, Universidad de Holguín.

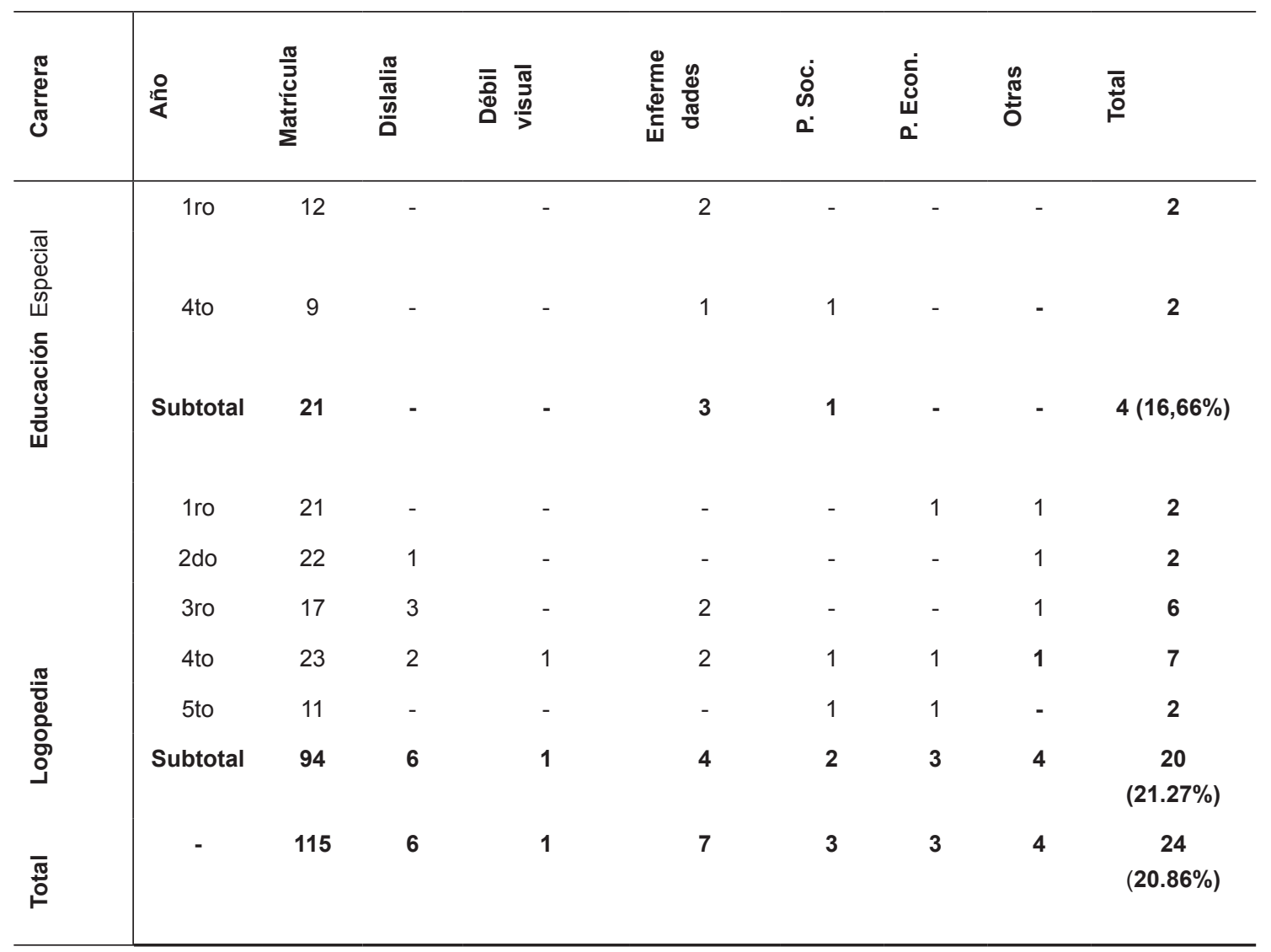

\section{Alumnos con necesidades educativas especiales, Universidad de Holguín.}

La orientación educativa se estructura en cuatro etapas: 1) caracterización y diagnóstico que se realiza al inicio del curso escolar; 2) encuentro del profesor guía con cada alumno y la negociación de los fines a alcanzar y las vías a utilizar en la orientación; 3) ejecución de las actividades de orientación con un enfoque dinámico y 4) valoración de los resultados individuales y colectivos cuando es pertinente. Igualmente, el departamento de Psicología colabora mediante el Gabinete de orientación psicosocial, proyecto de investigación iniciado en 2014, con un enfoque interdisciplinar que ofrece servicios en las diferentes sedes. Al triangular la información obtenida de los métodos utilizados se obtiene que la orientación educativa abarca las líneas siguientes:

- Reflexiones sobre elementos del diagnóstico que ayuden al autoconocimiento, la metacognición y el descubrimiento de capacidades para el aprendizaje.

- Sugerencias sobre los métodos de estudio según los estilos de aprendizaje y los objetivos y contenido de las disciplinas.

- Consejos sobre la actividad de estudio como el uso del tiempo, la organización de sus condiciones y la concentración de la atención.
- Proposiciones sobre la búsqueda de trabajo por horas y días que aumente el presupuesto económico.

- Sugestiones para la elaboración de proyectos de vida a corto, mediano y largo plazo que incluyen la continuidad de estudio y la incorporación a un empleo.

- Promoción de una actitud emprendedora según las potencialidades en la actividad científica y de investigación.

- Gestiones de impresión de materiales para los alumnos con dificultades visuales.

- Consejos para el establecimiento de las relaciones con sus pares, la pareja, la familia, los profesores y la solución de problemas.

- Establecimiento de la ayuda en el gabinete logopédico del departamento que ofrece atención desde 1986 y facilitar la atención médica especializada cuando sea necesaria.

- Incorporación de apoyo socio-afectivo, psicológico, psicoterapia y dinámicas familiares y de grupo.

- Coordinación con profesores de materias básicas de la enseñanza media para superar lagunas en conocimientos precedentes. 
Tabla 2. Alumnos con necesidades educativas especiales, Universidad de Cienfuegos.

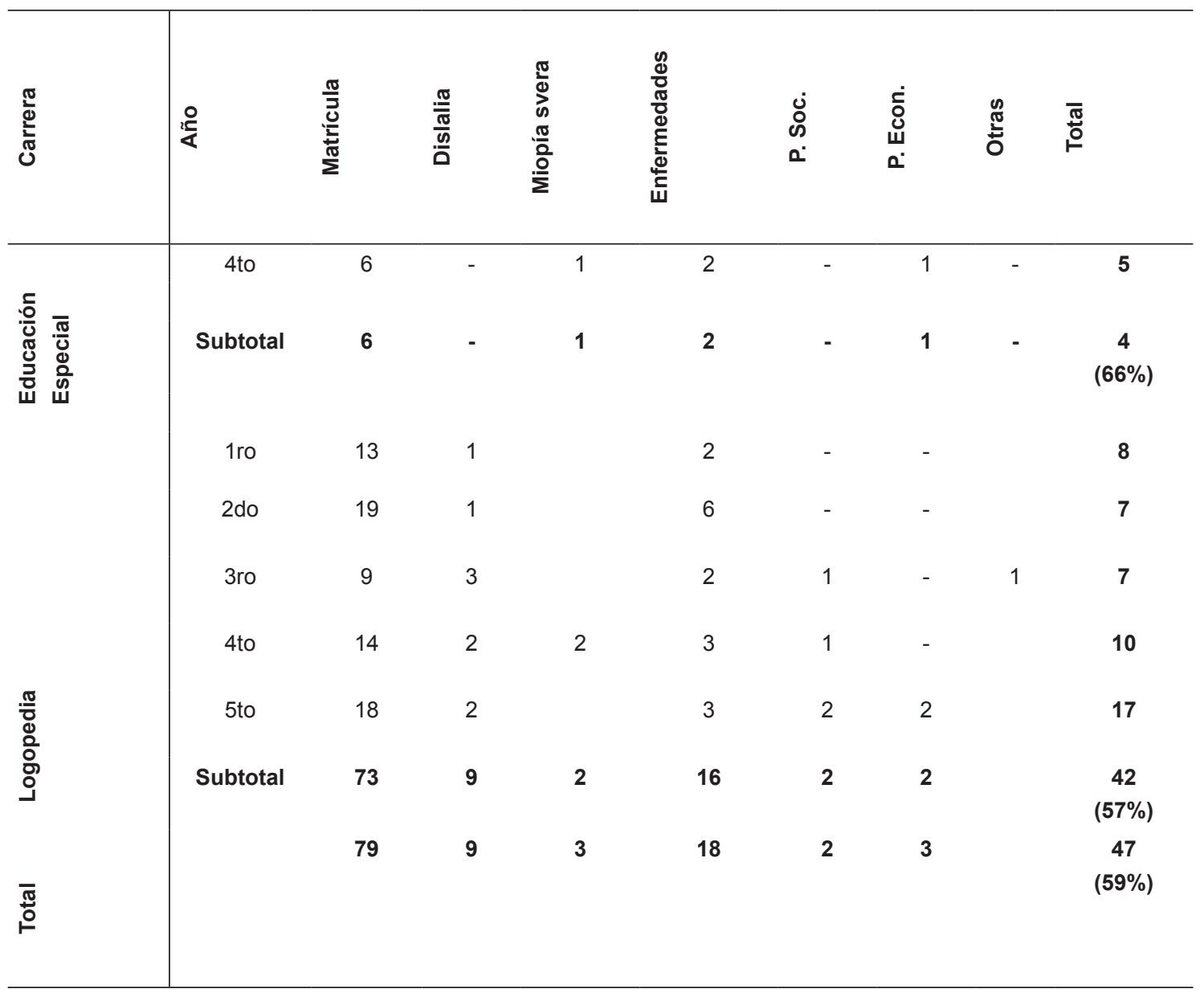

- Asesoría a los profesores sobre la aceptación de las diferencias, las estrategias y métodos de enseñanza y la evaluación del aprendizaje con un abordaje cualitativo, integrador y personalizado.

- Propuestas a los colectivos de disciplina,de año y a los directivos sobre variantes en el proceso de la práctica laboral y profesional.

- Promoción del diálogo entre directivos, profesores, alumnos y familia para establecer relaciones de cooperación y ofrecer apoyos individualizados: académicos, laborales, económicos y afectivos.

- Recomendación de informar a las instituciones de práctica y de empleo sobre las competencias de los estudiantes y los apoyos que requieren.

- Conversatorios en grupos de estudiantes y profesores con especialistas del territorio y de la universidad.

- Ajustes en las actividades de extensión universitaria ya sean deportivas o culturales.

- Creación de un espacio de colaboración con los padres y familiares que les permita apoyar a sus hijos respecto a:

- La toma de decisiones sobre la carrera elegida y los cambios de carrera.
- La inserción laboral con responsabilidad.

- La decisión de crear una familia.

• El tránsito a la vida adulta.

Caso 2: La orientación educativa en la Universidad de Cienfuegos.

La Universidad de Cienfuegos (1979) cuenta con siete facultades, la de Ciencias Pedagógicas incluye el departamento de Educación Especial y Logopedia, integrado por 26 profesores, con doctorado (6) y maestría (14). Un profesor con sordera imparte la asignatura de Lengua de señas cubanas y dos con ceguera - un doctor y un master $3 / 4$ imparten la asignatura Sistema Braille/Orientación y Movilidad. La carrera de Educación Especial tiene entre sus egresados seis con sordera y uno con ceguera (un doctor y dos másteres), en la actualidad solo tiene estudiantes en cuarto año (6), de ellos cinco con necesidades educativas especiales. La matrícula de la carrera de Logopedia es de 73 estudiantes de primero a quinto años y 17 (16.92\%) con necesidades educativas especiales. (Ver Tabla 2) 
Los factores causales se relacionan con enfermedades: fiebre reumática (2), miopía severa (3), intolerancia a alimentos derivados de harina y lactosa (1), asma (6), epilepsia (1), enfermedad de Crhon (1), Síndrome de Madelum (1) y alergias (3); también, vulnerabilidad económica (3) y social dada por alcoholismo (3), así como timidez (1) y dislalias (9) $3 / 4$ sigmatismo tres y rotacismo seis $3 / 4$. Un grupo de 12 alumnos (no se recogen en la Tabla 2 pues crearía confusión en la suma total) presentan poco desarrollo cultural con dificultades en la lectura, la construcción gramatical, errores ortográficos, dislalias culturales y limitado desarrollo del vocabulario, los cuales requieren de ayuda para alcanzar una formación profesional competente.

\section{Alumnos con necesidades educativas especiales, Universidad de Cienfuegos}

La orientación educativa se estructura en tres etapas: 1) la caracterización grupal e individual en cada brigada; 2) la elaboración de una estrategia educativa general, donde cada asignatura despliega las estrategias curriculares y,3) la ejecución de la estrategia. Se realiza una preparación del claustro para la orientación individual y grupal, y se utiliza como documento guía el material: La orientación educacional y la facilitación del desarrollo desde el rol profesional del maestro (Del Pino y Recarey, 2005). La orientación educativa se dirige a las líneas siguientes:

- Organización de un curso de diplomado para el claustro sobre orientación educativa que incluye vías de la ayuda logopédica, psicológica y pedagógica a utilizar en el proceso de enseñanza y aprendizaje.

- Consejos a los padres sobre cómo apoyar a sus hijos en los estudios universitarios, las relaciones interpersonales y la preparación para la vida laboral.

- Compartir la información del diagnóstico para brindar apoyo socio-afectivo, fortalecer el conocimiento de sí mismos, sus motivaciones, necesidades y proyectos de vida personal y profesional.

- Coordinación con un profesor de español para un entrenamiento en habilidades comunicativas (escuchar, hablar, leer y escribir), el enriquecimiento del léxico (sinonimia y antonimia),el ritmo y la fluidez en la lectura oral y la construcción de textos orales y escritos.

- Instrucción sobre métodos de estudio y organización del tiempo, tanto general como para materias específicas.

- Organización de la ayuda logopédica para lo cual se crea un gabinete con profesores de la carrera que ofrece atención a toda la comunidad universitaria.

- Sensibilización de claustro docente y directivos sobre las particularidades de los alumnos y vías de ayuda para su formación y egreso exitoso.

- Realización de actividades académicas y extensionistas en instituciones de apoyo con científicos y personas públicas: Centro de Referencia Latinoamericano para la Educación Especial, Instituto Pedagógico Latinoamericano y Caribeño, Ministerio del Interior, Ministerio de Salud Pública, Centro de Rehabilitación Neurológica, Asociación de Pedagogos de Cuba, Asociación Nacional de Sordos, Asociación Nacional de Ciegos y Asociación de Limitados Físico Motores.

- Establecimiento de un tutor responsable por el desarrollo académico y el entrenamiento para participaren eventos desde nivel departamental hasta internacional.

- Adiestramiento para mejorar la competencia comunicativa mediante la cooperación en actividades de las escuelasy la comunidad con presentaciones de libros, té literario y dramatizaciones.

- Enriquecimiento de la cultura general con actividades en la comunidad de: promoción de salud (Lucha antidroga, Sexualidad responsable, Comunicación positiva, Prevención de epidemias), identificación de personas con necesidades educativas especiales en la comunicación, trabajo voluntario (limpieza de playas) y divulgación de normas de comportamiento (en ómnibus y espaciossociales).

\section{Discusión}

El modelo de inclusión en la educación superior en Cuba tiene un componente teórico, integrado por las dimensiones y procesos (Pérez, 2015). A su vez, posee un componente mediador para introducir en la práctica sus postulados que es la orientación educativa.

Del estudio de caso realizado se teorizan un conjunto de regularidades que se instituyen en características de la orientación educativa, para los alumnos con necesidades educativas especiales en la educación superior en Cuba. Para ello se enfatiza, que bajo este concepto se incluyen tanto, el alumno con discapacidad como otros, que por diversos factores de tipo biológico, social y psicológico, requieren de una colaboración específica para superar las contradicciones del desarrollo y los problemas de la vida, desde el proceso formativo universitario.

La primera particularidad de esencia es que constituye una actividad educativa que integra los agentes educativos de la institución en un sistema único de influencias. A su vez, se organiza como una relación de ayuda en la formación de la personalidad que tiene como núcleo el concepto de tendencia hacia el futuro (Vigotsky, 1995) o sea el desarrollo de las potencialidades de cada persona.

Esta particularidad enlaza con la teoría de la compensación formulada por Vigotsky (1995), como proceso de construcción y desarrollo de equilibrio de la personalidad, mediante la reorganización de las funciones para superar las dificultades y, que está integrada por tres componentes. El primero es el potencial de compensación, dado por la subjetividad de cada persona que origina procesos dominantes de la psiquis. El segundo es el fondo de compensación que reside en las funciones biológicas conservada. Por último, las condiciones de compensación que se relacionan con la organización del escenario social. 
La orientación educativa se estructura en tres etapas generales aunque puede tener otras más detalladas en las diferentes instituciones del país, estas son: la caracterización y diagnóstico, la proyección de las actividades de ayuda y la ejecución con un proceso sistemático de control y actualización.

Asimismo, se modela con un enfoque holístico, participativo y personalizado en los diferentes escenarios en que estos jóvenes participan en la institución universitaria: la clase, las actividades de estudio, la investigación, la actividad laboral y la labor extensionista, interrelacionada con los servicios especializados. Su centro se encuentra en la comunidad académica de año con el profesor guía como coordinador, con lo que se crea un espacio educativo idóneo para satisfacer las necesidades de cada alumno.

Esta actividad no se circunscribe al contexto de la institución universitaria, por lo cual se dirige a tres agentes esenciales para la educación en general:

Primero, a los alumnos, para apoyarles en la organización de la actividad de aprendizaje, la practica laboral y profesional, la resolución de problemas y los proyectos de vida personales y profesionales.

Segundo, a los profesores, como vía para asistirles en la atención a la diversidad del alumnado, lo cual incluye la modelación didáctica del proceso docente, las exigencias de la investigación y la proyección de su inclusión laboral.

Tercero, a los padres, que les auxilia para que apoyen a sus hijos en la toma de decisiones y el tránsito a la vida adulta y laboral con responsabilidad.

En esta labor de orientación es determinante el agente educativo profesor, ya que su actividad formativa tiene implicaciones directas en el éxito del proceso educativo. El profesor es el principal mediador en el aprendizaje y en la medida que el alumnado es más diverso, requiere de preparación para satisfacer las demandas de sus alumnos.

La orientación al profesor no se circunscribe en sugerirle herramientas para el currículo sino que junto con ello se proyecta la modificación de actitudes que les permita tener en cuenta la pluralidad de desarrollos de sus alumnos. De manera que se pueda colocar en el lugar de los otros y buscar todas las vías que puedan ayudar y apoyar a los alumnos.

La orientación educativa desenvuelve conceptos básicos de la psicología histórico cultural, principalmente: la situación social del desarrollo en el proceso de caracterización y diagnóstico; la zona de desarrollo próximo al implementar la colaboración y la unidad del desarrollo, en la ejecución y valoración de los resultados individuales con lo que se da paso a otro nivel del desarrollo y la propuesta de nuevas metas y vías. La utilización del método dialéctico ayuda a comprender la fuente del desarrollo en fuerzas que se oponen y, que la orientación educativa contribuye a encauzar y, a su vez, revela el carácter y la dinámica del proceso.

\section{Conclusiones}

La educación superior está retada a cambiar y mientras las políticas evolucionan a un ritmo desventajoso, quienes implementan los procesos formativos en las instituciones están transformando sus prácticas. De esta manera, modelar sistemas educativos más inclusivos "exige un fuerte compromiso de trabajar hacia una sociedad más justa, equitativa y pacífica" (Unesco, 2008, p.5).

Los documentos normativos en la educación superior cubana se conciben como si el alumnado fuese una masa homogénea y, contiene referencias aisladas a la inclusión de los jóvenes con necesidades educativas especiales. Sin embargo, el contexto humanista general promueve prácticas inclusivas que introducen cambios en todo el sistema proporcionando oportunidades ajustadas al desarrollo cada alumno, como "una manera de asegurar que la Educación Para Todos signifique realmente todos" (Unesco, 2008, p. 6).

Del estudio de casos realizado en dos universidades cubanas se deriva que la orientación educativa para la inclusión de los alumnos con necesidades educativas especiales en la educación superior, se sustenta en la articulación de postulados de la psicología histórico cultural, la concepción pedagógica de las necesidades educativas especiales y el modelo social de la discapacidad. Es por ello que beneficia no solo a los alumnos con discapacidad, sino que contribuye a satisfacer las demandas de cada alumno que precisa de ayudas para permanecer en la universidad, egresar con éxito e iniciar la vida laboral.

\section{Referencias}

Cabral, L.S.A; Mendez, E.G; De Anna, L. (2015). Orientação académica e profissional dos estudantes com deficiência nas universidades italianas. Revista Ibero-Americana de estudos em Educação, 10 (n. esp), 615-629.

Calviño, V.M. (2000). La orientación psicológica. Esquema referencial de alternativa múltiple. La Habana: Científico-Técnica.

Chernousova, L.N. (2008). Estimulación de la comunicación verbal en escolares con Necesidades Educativas Especiales en el desarrollo general del lenguaje. Disertación Doctoral, Instituto Superior Pedagógico, Holguín-Cuba.

Collazo, D.B.; Puentes, A. M. (1992). La orientación en la actividad pedagógica. La Habana: Pueblo y Educación.

Cuba (2010). Circular Mined-Mes/2010. Sobre el tratamiento a los jóvenes discapacitados para estudiar en la Educación Superior. La Habana.

Cuba. Oficina Nacional de estadísticas e Información [ONEI] (2014). Informe Nacional del Censo de población y vivienda de Cuba de 2012. Publicado con la colaboración del: Fondo de Población de las Naciones Unidas, UNFPA.

Del Pino, J.L.; Recarey, S. (2005). La orientación educacional y la facilitación del desarrollo desde el rol profesional del maestro. Material básico de la Maestría en Ciencias de la Educación, 
Soporte Digital.

Domínguez, M.I. (2016). Educación superior en Cuba e inclusión social de las juventudes. Nómadas, 44, 83-103.

López, M.M. (2011). Barreras que impiden la escuela inclusiva y algunas estrategias para construir una escuela sin exclusiones. Innovación Educativa, 21, 37-54.

ONU (2015). Transformar nuestro mundo: la Agenda 2030 para el Desarrollo Sostenible. Recuperado: 14 mar. 2017. Disponível: http://unctad.org/meetings/es/SessionalDocuments/ares70d1_ es.pdf.

Pérez, E.A. (2015). La educación inclusiva en la Educación Superior: relato del contexto universitario cubano.Revista Ibero-Americana de estudos em Educação, 10 (n. esp), 583-597.

Pla, M. (1999). El rigor en la investigación cualitativa. Revista de Atención Primaria, 24, 295-300.

Pérez-Cruz, F.J. (2011). La Campaña Nacional de Alfabetización en Cuba. Revista Varona, 53, 10-23. Recuperado: 4 ago. 2017. Disponível: http://www.redalyc.org/pdf/3606/360635575003.pdf

Resolución Ministerial no 132/2004(2004). Reglamento de Educación de Postgrado de la República de Cuba. La Habana: MES. Recuperado: 22 jan. 2017. Disponível: http://instituciones.sld. cu/ucmh/files/2012/01/RM-132-04-Reglamento-de-la-Educ-dePosgrado.pdf.

Resolución Ministerial $n^{\circ} 120 / 2010$ (2010) o Reglamento de organización docente de la educación superior. La Habana: MES.
Recuperado: 22 jan. 2017. Disponível: http://files.sld.cu/sccs/ files/2010/09/reglamento-org-docente-mes-res-120-2010.pdf

Taylor, S.J.; Bogdan, R. (1996). Introducción a los métodos cualitativos de investigación. La búsqueda de significados (3ra reimpresión). Barcelona: Paidós.

Unesco (2008). Conferencia internacional de educación, La educación inclusiva: el camino hacia el futuro.Recuperado: 14 mar. 2017. Disponível: http://www.ibe.unesco.org/fileadmin/user_upload/ Policy_Dialogue/48th_ICE/General_Presentation-48CIE-4_ Spanish_.pdf.

Unesco (2015).Informe de Seguimiento de la Educación Para Todos en el Mundo 2015. Recuperado: 6 mar. 2017. Disponível: http:// unesdoc.unesco.org/images/0023/002324/232435s.pdf.

Vigotsky, L.S. (1982). Pensamiento y Lenguaje. La Habana: Pueblo y Educación.

Vigotsky, L.S. (1987). Obras completas. Tomo III. Historia del desarrollo de las funciones psíquicas superiores. La Habana: Editorial Científico-Técnica.

Vigotsky, L.S. (1995). Obras Completas, Tomo V. Defectología. Habana: Pueblo y Educación.

License information: This is an open-access article distributed under the terms of the Creative Commons Attribution License (type CCBY), which permits unrestricted use, distribution and reproduction in any medium, provided the original article is properly cited.

Recebido em: 22/08/2017 Aprovado em: 07/11/2017

\section{Sobre as autoras}

Elsie Alejandrina Pérez Serrano (elsieperezserrano@gmail.com)

Fonoaudióloga, Maestría en Educación Especial y Doctora en Pedagogía. Profesora Titular del Departamento de Desarrollo Local de la Universidad de Holguín, Cuba. Cursa posdoctorado en el programa de Postgrado en Educación de la Universidad Federal de São Carlos (becaria CNPq) e integrante del Núcleo de Estudios e Investigaciones sobre la Escuela de Vigotsky - NEEVY y del Núcleo de Investigación sobre la Enseñanza Superior - NUPES. Miembro del Grupo de Estudios de Géneros, de la Cátedra de Estudios de la Mujer y de la Cátedra de Antropología de la Universidad de Holguín y presta asesoría pedagógica a redes de enseñanza. https://orcid.org/0000-0001-8272-275X

Elsa María Hernández Ochoa (emhernandez@ucf.edu.cu)

Fonoaudióloga, graduada del Programa de estudios Avanzados: Doctorado en Diversidad, currículo y educación de La Universidad de Girona España, Doctora en Pedagogía. Fue fonoaudióloga de escuela especial para alumnos con discapacidad mental durante 10 años. Profesora Titular del Departamento de Educación Especial, de La Facultad de Educación de la Universidad de Cienfuegos, Cuba. Coordinadora del Programa de Graduación en Logopedia (Fonoaudiología) y de La Especialización en Logopedia. Asesora metodológica del Departamento de Formación metodológica de la Pro - Rectoría Docente, de La Universidad de Cienfuegos. Miembro de La Comisión Nacional de Carrera en Logopedia y Consultor Científico de La Asociación de Pedagogos de Cuba. https://orcid.org/0000-0002-1969-1601 